\title{
CYLINDRICAL PENNING TRAPS WITH ORTHOGONALIZED ANHARMONICITY COMPENSATION *
}

\author{
GERALD GABRIELSE and F. COLIN MACKINTOSH \\ Department of Physics, University of Washington, Seattle, WA 98195 (U.S.A.)
}

(First received 13 June 1983; in final form 14 October 1983)

\begin{abstract}
Compensated Penning traps with cylindrical ring and compensation electrodes and flat endcaps are considered as alternatives to high precision traps with hyperbolic ring and endcap electrodes. Cylindrical and flat electrodes can be more easily and precisely constructed, especially for the very small traps which are desirable for ion trapping. They are easily studied since Laplace's equation can be solved by a series expansion which is discussed in most electricity and magnetism textbooks. A central new result is that a judicious choice of the height-to-diameter ratio for a cylindrical trap makes the axial oscillation frequency of a trapped particle independent of adjustments in the compensation potential, just as has been recently proposed for hyperbolic traps. Other properties of such orthogonalized cylindrical traps appear to be adequate for particle trapping so that properly designed cylindrical traps are promising alternatives for high precision work, ready for laboratory testing. Whether or not cylindrical traps will be able to replace hyperbolic traps entirely for the highest precision work, a major point of this paper is that orthogonalized traps can be built with any reasonable electrode geometry. Special access traps with orthogonalized anharmonicity compensation are thus completely feasible, though numerical calculations will be generally required for their design.
\end{abstract}

\section{INTRODUCTION}

A single electron was first trapped in a Penning trap 10 years ago [1]. Subsequent progress [2] led to measurements of the magnetic moments of both the electron [3] and positron [4] to accuracies within $5 \times 10^{-11}$. The measurements of the magnetic moment anomalies are the most stringent tests of quantum electrodynamics which has recently been used to calculate the anomalies to the order $\alpha^{4}$ [5]. Comparison of the electron and positron magnetic moments provides an unprecedented test of the invariance of the

* Supported by the National Science Foundation. 
electron/positron system under CPT (symmetry under charge conjugation, parity, and time reversal). An experiment now underway has already produced the most accurate proton/electron mass ratio with great improvements expected when a single proton is trapped [6]. Another experiment is underway to improve the measurement of the magnetic moment of the electron to $10^{-12}$ or better [7].

All of the precision experiments mentioned were made in compensated Penning traps with hyperbolic ring and endcap electrodes [8]. The electrostatic properties of such traps [9] and the effect of radiofrequency potentials on the endcaps [10] have already been investigated via relaxation calculations. These calculations showed that a judicious choice of hyperbolic endcap and ring electrodes makes the axial oscillation frequency of a trapped particle independent of changes in the compensation potential [9]. A better understanding of the electrostatic properties of hyperbolic traps suggested that the ratio of minimum distances between the endcap and ring electrodes was much more important than the hyperbolic contours themselves. It seemed that hyperbolic electrodes might be less necessary for precision work than had been earlier assumed.

Compensated Penning traps with cylindrical ring and compensation electrodes and flat endcap electrodes are considered here as alternatives to hyperbolic Penning traps. Uncompensated cylindrical traps have long been used for particle containment when only a crude quadrupole potential is required [11], most commonly in ion pumps, for example. We investigate here the possibility of improving the quadrupole potential within such traps by the addition of compensation electrodes and by a judicious choice of trap dimensions so that they can be used for high precision experiments of the type mentioned above. Cylindrical electrodes have the important practical advantage that they can be machined to much greater accuracy in much less time than can hyperbolic electrodes, especially for the very small traps which may be desirable for ion trapping. The theoretical study of cylindrical traps is also much easier than the relaxation calculation used for hyperbolic traps. Laplace's equation is readily solved for a cylinder with flat ends using standard techniques discussed in most electricity and magnetism textbooks. The series solutions converge rapidly and can be evaluated with a programmable calculator.

The notation used (Sect. 2) is deliberately similar to that used earlier for hyperbolic traps [9] to facilitate a comparison of their properties. The general discussion of anharmonicity compensation is left to the earlier paper with only a brief summary provided in Sect. 2 . Two cylindrical traps are considered. The first (Fig. 1 and Sect. 3) has a single cylindrical ring elcetrode and two flat endcaps. It can only be mechanically tuned to reduce anharmonicities. The second (in Fig. 2 and Sect. 4) is the cylindrical analog of the 
successful hyperbolic traps. Compensation electrodes are included next to the endcaps and the potential on these is varied to reduce anharmonicities. A notable new result is that a judicious choice of the height-to-diameter ratio makes the axial oscillation frequency of a trapped particle independent of the compensation potential, just as occurs for the proposed orthogonalized hyperbolic trap design [9]. This is a considerable improvement over the hyperbolic traps used for the precision experiments mentioned above. Many other properties of cylindrical traps, including the axial damping by a resistor between the endcaps (Sect. 5) [10], are comparable with or superior to those for hyperbolic traps.

Perfect hyperbolic trap electrodes would certainly produce the desired quadrupole potential over a much larger trapping volume than is possible with the cylindrical geometry studied here. There is growing evidence, however, that the usable trapping volume within existing hyperbolic traps is limited by electrode misalignments and imperfections along with holes and slits in the electrodes (to admit particles and various radiofrequency and microwave drives). The extent to which this is true, and thus the extent to which a cylindrical trap can provide a comparable trapping volume, must now be tested experimentally. Whether or not cylindrical traps are capable of replacing hyperbolic traps for precision work, a major point of this paper is that orthogonalized, compensated Penning traps can be constructed for any reasonable electrode geometry. The orthogonalized configuration of hyperbolic electrodes has been determined earlier by a relaxation calculation [9]. The orthogonalized configuration of cylindrical electrodes is calculated here using well-known electrostatic techniques. Other electrode configurations, to permit special access for lasers or particle transfer, for example, are certainly possible. Determining the orthogonalized configuration will generally require a numerical calculation which is tedious but completely manageable.

\section{CYLINDRICAL PENNING TRAPS}

The two cylindrical Penning traps to be considered are represented in Figs. 1 and 2. Both traps are symmetric under rotations about the $z$ axis (which is aligned along a homogeneous magnetic field) and under reflections across the $x y$ plane. Flat grounded endcaps at potential $V_{0} / 2$ are perpendicular to the $z$ axis and are located at $z= \pm z_{0}$. Stacked cylindrical ring electrodes of radius $\rho_{0}$ complete the traps. For both configurations, the potential of the central electrode (called the ring) is $-V_{0} / 2$ so that $V=0$ is midway between the potentials of the endcaps and ring electrodes. This same convention was used for hyperbolic traps [9]. Compensation electrodes at potential $V_{\mathrm{c}}$ and height $\Delta z_{\mathrm{c}}$ are inserted next to the flat endplates in the trap 

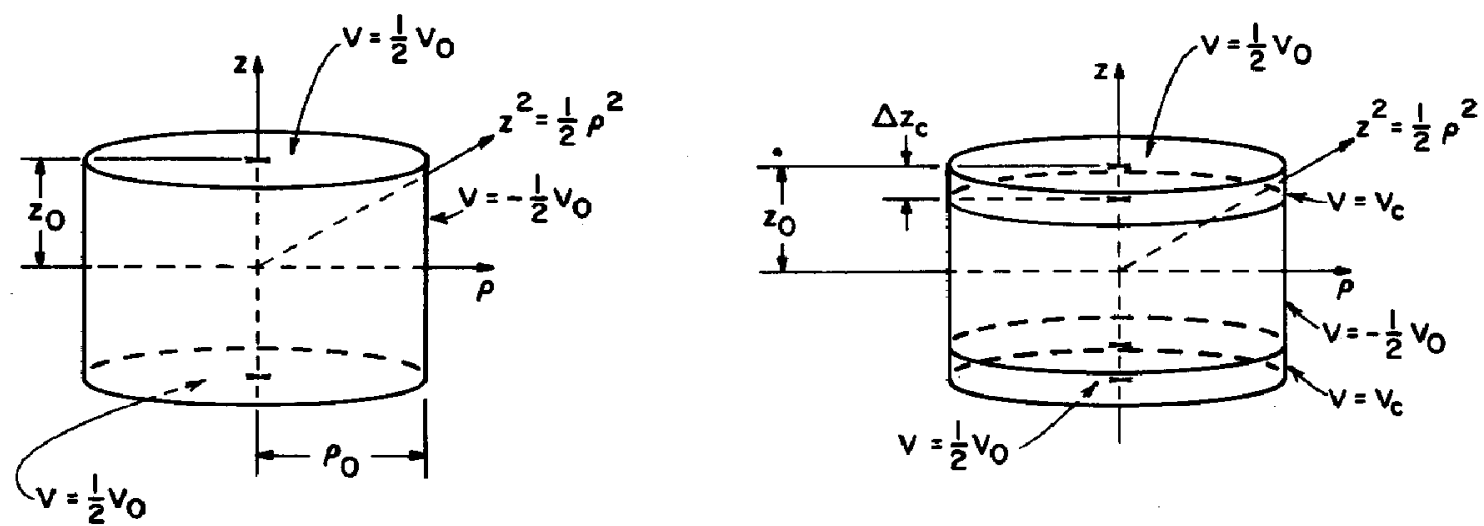

Fig. 1. Simple Penning trap with flat endcaps at $z= \pm z_{0}$ with potential $V_{0} / 2$ and a single, cylindrical ring electrode of radius $\rho=\rho_{0}$ and potential $-V_{0} / 2$. The asymptote $z^{2}=\frac{1}{2} \rho^{2}$ for the desired quadrupole potential is also shown. To tune out anharmonicities, $z_{0}$ could be slightly adjusted during an experiment.

Fig. 2. Electrically compensated cylindrical trap.

of Fig. 2. The compensation potential $V_{c}$ is varied to improve the potential near the center of the trap.

Complete knowledge of the potential $V$ within these traps is generally not required because particles are typically trapped near the center of the trap. In this region (at position $[r, \theta . \phi]$ in spherical coordinates with $r \ll d$ ) the potential can be expanded in a familiar way in even powers of the small ratio $r / d$ multiplied by even order Legendre polynomials $P_{k}(\cos \theta)$.

$$
V=\frac{1}{2} V_{0} \sum_{k_{\mathrm{cren}}=0}^{\infty} D_{k}\left[\frac{r}{d}\right]^{k} P_{k}(\cos \theta)
$$

The distances are scaled by the characteristic trap dimension $d$ defined in the earlier paper [9] by

$d^{2}=\frac{1}{2}\left[z_{0}^{2}+\frac{1}{2} \rho_{0}^{2}\right]$

The $D_{k}$ expansion coefficients are exact counterparts of the $C_{k}$ used for hyperbolic traps except that $D_{2}$ corresponds to $1+C_{2}$. For an ideal quadrupole trap, all the $D_{k}$ vanish except for $D_{2}$.

The lowest order coefficients in expansion (1) are most important for particle trapping since $r \ll d$. These are discussed in detail in ref. 9. To summarize, $D_{0}$ is unobservable and is thus ignored. The second coefficient, $D_{2}$, relates the axial oscillation frequency $\omega_{z}$ for the trapped particle of charge $q$ and mass $m$ to the trapping potential $V_{0}$.

$\omega_{z}^{2}=\frac{q V_{0}}{m d^{2}} D_{2}$ 
Higher-order $D_{k}$ quantify trap anharmonicities. Certainly, $D_{4}$ is the most important and its most important consequence is a very undesirable amplitude-dependent shift in $\omega_{z}$ [12].

$$
\frac{\Delta \omega_{z}}{\omega_{z}}=\frac{3}{2} \frac{D_{4}}{D_{2}} \frac{E_{z}}{m \omega_{z}^{2} d^{2}}
$$

The ratio of energies involves the axial excitation energy $E_{z}$ and the axial well depth $m \omega_{z}^{2} d^{2}$ and is typically very small. The consequences of $D_{6}$ are typically smaller still and can be included by replacing $D_{4}$ in Eq. (4) with

$\tilde{D}_{4}=D_{4}+\frac{5}{2} D_{6} \frac{E_{z}}{m \omega_{z}^{2} d^{2}}$

Traps are tuned to minimize amplitude-dependent shifts and/or broadening of the axial resonance. Uncompensated traps with hyperbolic electrodes $[13,14]$ have been constructed with $\left|\tilde{D}_{4}\right| \approx 10^{-2}$ to $10^{-3}$. Compensated traps have produced $\left|\tilde{D}_{4}\right|<10^{-4}$ when carefully tuned.

To achieve very low values of $D_{4}$, it is important to make changes, $\Delta D_{4}$, while an experiment is in progress. For the mechanically tuned trap of Fig. 1, this is accomplished by adjusting $\rho_{0} / z_{0}$. For the electrically compensated trap of Fig. 2, this is accomplished by adjusting the compensation potential, $V_{\mathrm{c}}$. Such adjustments, however, will generally also produce a change $\Delta D_{2}$ (and hence a highly undesirable change in $\omega_{z}$ ) as well. A quality factor $\gamma$ was defined earlier to quantify this trade-off [9]. For our purposes

$\gamma \equiv \frac{\Delta D_{2}}{\Delta D_{4}}$

with $\Delta D_{2}$ and $\Delta D_{4}$ both resulting from the same anharmonicity adjustment. Ideally, $\gamma$ would vanish so that $\omega_{z}$ is completely unaffected by anharmonicity adjustments. The orthogonalized hyperbolic trap proposed in ref. 9 and its cylindrical counterpart in Sect. 4 allow the ideal value of $\gamma=0$ to be realized in principle. As a reference value, all of the precision measurements referred to earlier were done with asymptotically symmetric Penning traps with hyperbolic electrodes and electrical frequency compensation for which $\gamma \approx$ 0.56 .

\section{MECHANICALLY COMPENSATED TRAP}

The simplest cylindrical Penning trap in Fig. 1 has only a single cylindrical ring electrode. A series solution for the potential within this trap

$$
V=V_{0}\left[\frac{1}{2}+\sum_{n=0}^{\infty} A_{n} J_{0}\left[i k_{n} \rho\right] \cos \left[k_{n} z\right]\right]
$$


is readily obtained by standard techniques which are discussed in most textbooks. The constants

$k_{n}=\frac{\left[n+\frac{1}{2}\right] \pi}{z_{0}}$

are defined so that the cosine factors (which are even under the reflections $z \rightarrow-z$ ) vanish on the endcaps. The zero-order Bessel function suffices because of invariance under rotations about the $z$ axis. Despite its imaginary argument, this function is real and easily evaluated from the well-known expansion

$J_{0}(i x)=\sum_{j=0}^{\infty} \frac{1}{(j !)^{2}}\left[\frac{x}{2}\right]^{2 j}$

Inverting Eq. (7) by integrating over the trap electrodes in the standard way yields

$A_{n}=4 \frac{(-1)^{n+1}}{(2 n+1) \pi J_{0}\left[i k_{n} \rho\right]}$

which, along with Eq. (7) comprises a complete and unique solution to Laplace's equation for the boundary conditions in Fig. 1.

For particle trapping, we are interested in the coefficients $D_{k}$ for expansion (1) for small $r / d$. The $D_{k}$ of Eq. (1) can be simply related to the $A_{n}$ of Eq. (7) by evaluating both expansions on the $z$ axis. Equating coefficients of $z^{k}$ yields (for $k>0$ and even)

$D_{k}=2 \frac{(-1)^{k / 2}}{k !}\left[\frac{\pi}{2}\right]^{k}\left[\frac{d}{z_{0}}\right]^{k} \sum_{n=0}^{\infty}(2 n+1)^{k} A_{n}$

Substituting the $A_{n}$ for the simple trap of Fig. 1 yields (again for $k>0$ and even)

$D_{k}=\frac{(-1)^{k / 2}}{k !} \frac{\pi^{k-1}}{2^{k-3}}\left[\frac{d}{z_{0}}\right]^{k} \sum_{n=0}^{\infty} \frac{(-1)^{n+1}(2 n+1)^{k-1}}{J_{0}\left[i k_{n} \rho_{0}\right]}$.

Observe that the $D_{k}$ are functions of only $\rho_{0} / z_{0}$ as might be expected and that the series converges rapidly since, for large arguments, $J_{0}(i x)$ goes as $\mathrm{e}^{x} /(2 \pi x)^{1 / 2}$.

The series expression for $D_{k}$ in Eq. (12) can be evaluated with a hand calculator. Figure 3 shows $D_{2}, D_{4}$ and $D_{6}$ as functions of $\rho_{0} / z_{0}$. Observe that $D_{4}$ vanishes at

$\rho_{\mathrm{o}} \approx 1.203 z_{0}$

For this choice, $D_{2} \approx 1.126$ and $D_{6} \approx-0.095$. Achieving small values of $D_{4}$ in a laboratory trap will be much more likely if $D_{4}$ can be tuned during an 

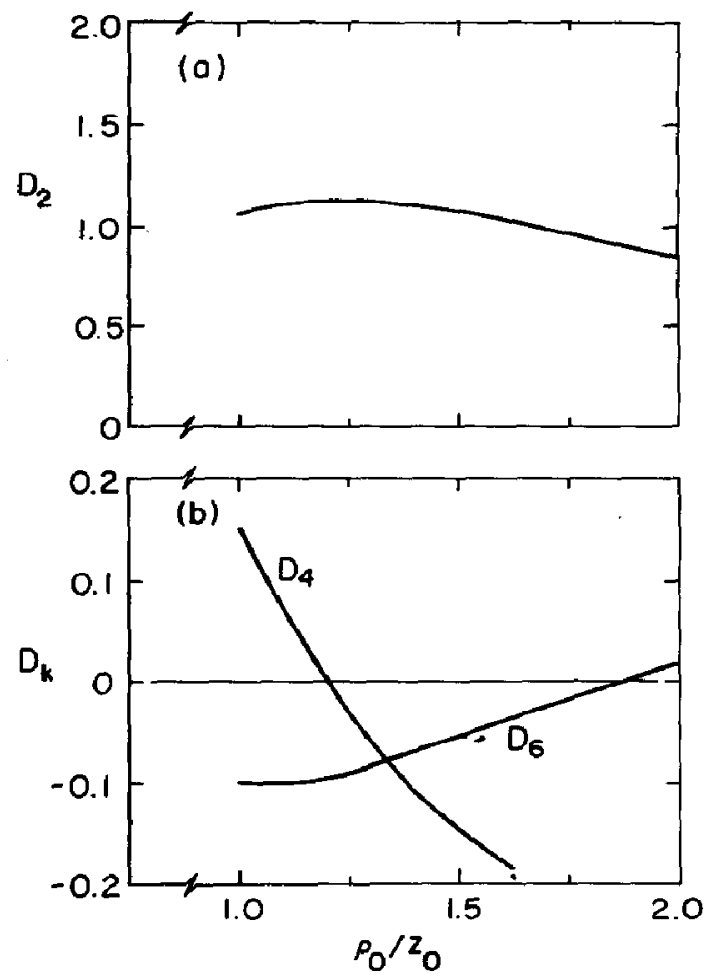

Fig. 3. Lowest order $D_{k}$ for the simple trap of Fig. 1 as a function of $\rho_{0} / z_{0}$.

experiment. The most feasible adjustment for the trap of. Fig. 1 is a small change in $\rho_{0} / z_{0}$ [from the value in Eq. (13) which makes $D_{4}=0$ for a perfect trap] by sliding an endcap in or out of the ring slightly. Such mechanical anharmonicity compensation is characterized by the derivative

$\frac{\partial\left[D_{4} / D_{2}\right]}{\partial\left[z_{0} / \rho_{0}\right]} \approx 0.81$

and by the very respectable quality factor

$\gamma=-0.095$

because $D_{2}$ is near a maximum. Notice, however, that to reduce $\left|\tilde{D}_{4}\right|$ below $10^{-4}$ (as has been done in electrically compensated traps with hyperbolic electrodes) requires adjusting $z_{0} / \rho_{0}$ to within $10^{-4}$ of the optimal value, which makes $D_{4}=0$. This is a very rigorous requirement, especially considering the high vacuum and low temperature environments often used for precision experiments.

\section{ELECTRICALLY COMPENSATED CYLINDRICAL TRAPS}

The electrically compensated trap of Fig. 2 is the cylindrical counterpart of the highly successful hyperbolic traps. Anharmonicity compensation (i.e. 

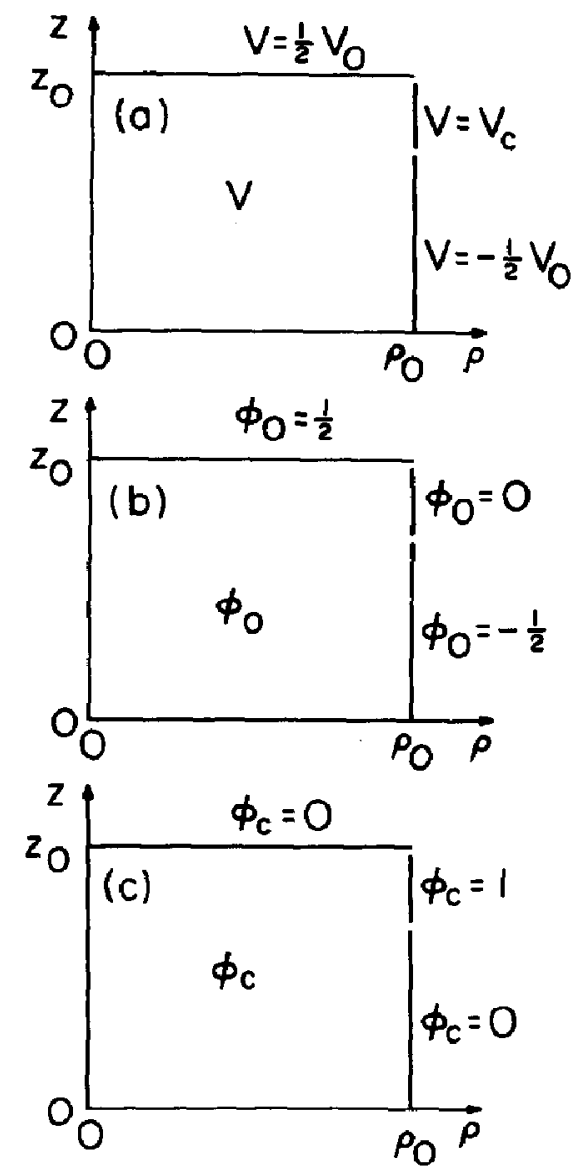

Fig. 4. Boundary conditions for the electrically compensated cylindrical trap of Fig. 2 in (a) and for the solutions to Laplace's equation $\phi_{0}$ and $\phi_{c}$ in (b) and (c). Symmetry under rotations about the $z$ axis and under the reflection $z \rightarrow-z$ are assumed.

the reduction of the magnitude of $D_{A}$ ) is accomplished while an experiment is in place by tuning the compensation potential $V_{c}$. The properties of the cylindrical traps are compared with the properties of the hyperbolic traps. This is possible because the properties of the latter have been established quantitatively by a relaxation calculation [9]. To facilitate the comparison, the notation in the next several paragraphs is exactly analogous to that discussed in more detail in ref. 9.

The boundary conditions for the trap of Fig. 2 [repeated in Fig. 4 (a) for $\rho \geqslant 0$ and $z \geqslant 0]$ are a superposition of the boundary conditions in Fig. 4(b) and (c). Solutions to Laplace's equation, $\phi_{0}$ and $\phi_{c}$, are defined by these latter boundary conditions and by the invariance of these solutions under the reflection $z \rightarrow-z$. Thus the potential within the trap is given by

$V=V_{0} \phi_{0}+V_{c} \phi_{c}$ 
and, as a result, each $D_{k}$ in the expansion (1) for small $r / d$ is again the sum of two terms

$D_{k}=D_{k}^{(0)}+\left[V_{0} \frac{\partial D_{k}}{\partial V_{c}}\right] \frac{V_{c}}{V_{0}}$

The $D_{k}^{(0)}$ pertain to a compensation potential $V_{c}=0$ which is midway between the endcap and ring potentials. A non-zero compensation potential $V_{\mathrm{c}}$ produces the second term which can be used to cancel the first and thus eliminate a troublesome $D_{k}$, typically $D_{4}$. The constants $V_{0} \partial D_{k} / \partial V_{c}$ are called the tunabilities for $D_{k}$ because they quantify a trap's ability to tune out the $D_{k}$.

It is important to realize that the dimensionless constants $D_{k}^{(0)}$ and $V_{0} \partial D_{k} / \partial V_{c}$ are actually properties only of the trap geometry and are independent of $V_{0}, V_{c}$ and $d$. These constants are, in fact, the expansion coefficients for the expansion of $\phi_{0}$ and $\phi_{c}$ in $r / d$ with

$\phi_{0}=\frac{1}{2} \sum_{k_{\text {even }}=0}^{\infty} D_{k}^{(0)}\left[\frac{r}{d}\right]^{k} P_{k}(\cos \theta)$

and

$\phi_{\mathrm{c}}=\frac{1}{2} \sum_{k_{\mathrm{even}}=0}^{\infty}\left[V_{0} \frac{\partial D_{k}}{\partial V_{\mathrm{c}}}\right]\left[\frac{r}{d}\right]^{k} P_{k}(\cos \theta)$

The earlier paper contains a more detailed discussion of these expansions [9].

Both $\phi_{0}$ and $\phi_{\mathrm{c}}$ can be solved analytically using the standard techniques reviewed in Sect. 3. Solving for $\phi_{0}$ yields (for $k>0$ and even)

$D_{k}^{(0)}=\frac{(-1)^{k / 2}}{k !} \frac{\pi^{k-1}}{2^{k-3}}\left[\frac{d}{z_{0}}\right]^{k} \sum_{n=0}^{\infty} \frac{(-1)^{n+1}(2 n+1)^{k-1} \cos ^{2}\left[\frac{1}{2} k_{n} \Delta z_{\mathrm{c}}\right]}{J_{0}\left[i k_{n} \rho_{0}\right]}$

Solving for $\phi_{\mathrm{c}}$ yields (again for $k>0$ and even)

$V_{0} \frac{\partial D_{k}}{\partial V_{c}}=\frac{(-1)^{k / 2}}{k !} \frac{\pi^{k-1}}{2^{k-3}}\left[\frac{d}{z_{0}}\right]^{k} \sum_{n=0}^{\infty} \frac{(-1)^{n}(2 n+1)^{k-1} 2 \sin ^{2}\left[\frac{1}{2} k_{n} \Delta z_{c}\right]}{J_{0}\left[i k_{n} \rho_{0}\right]}$

Notice that, in the limit, the compensation electrode goes to zero height (i.e. $\Delta z_{\mathrm{c}} \rightarrow 0$ ), the tunabilities $V_{0} \partial D_{k} / \partial V_{\mathrm{c}}$ vanish term by term and the $D_{k}^{(0)}$ become the $D_{k}$ in Eq. (12), which pertain to the simple trap of Fig. 1. More generally, the $D_{k}$ for the simple trap of Fig. 1 are equal to the sum of $D_{k}^{(0)}$ and $-1 / 2\left(V_{0} \partial D_{k} / \partial V_{c}\right)$ as must be by Eq. (17) when the compensation potential $V_{\mathrm{c}}$ is equal to the ring potential $-V_{0} / 2$.

The quality factor $\gamma$ of Eq. (6) is given for electrically compensated traps by [9]

$\gamma=V_{0} \frac{\partial D_{2}}{\partial V_{c}} / V_{0} \frac{\partial D_{4}}{\partial V_{c}}$ 


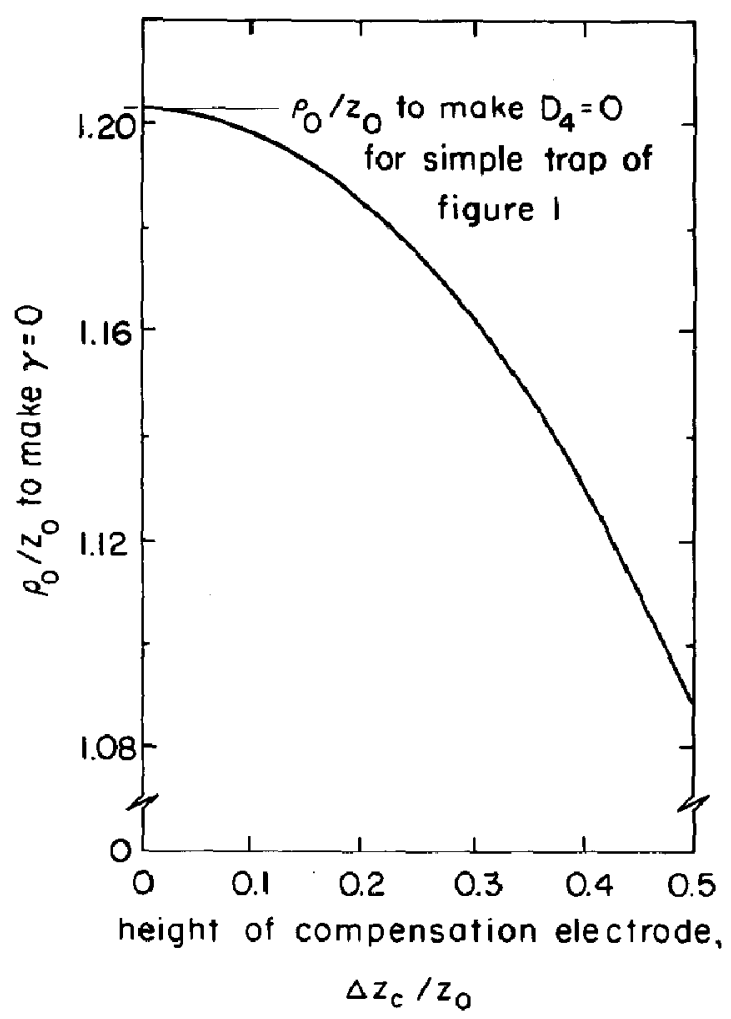

Fig. 5. The ratio $\rho_{0} / z_{0}$ required to make the axial oscillation frequency $\omega_{2}$ of a particle trapped in the trap of Fig. 2 independent of the compensation potential $V_{c}$ applied to a compensation electrode of height $\Delta z_{\mathfrak{c}}$.

Thus, $\gamma$ can be evaluated from Eq. (21) as a function of $\rho_{0} / z_{0}$ and $\Delta z_{\mathrm{c}} / z_{0}$. From Eq. (6), $\gamma=0$ means that the necessary adjustments of the compensation potential $V_{c}$, to tune out anharmonicities, produce no corresponding changes in the axial oscillation frequency $\omega_{z}$. The required $\rho_{0} / z_{0}$ is plotted in Fig. 5 as a function of $\Delta z_{\mathrm{c}} / z_{0}$. It changes by only $10 \%$ for $\Delta z_{\mathrm{c}} / z_{0}$ ranging from 0 to $1 / 2$. Notice that the ratio $\rho_{0} / z_{0}$ required to make $\gamma=0$ varies around the value

$\rho_{0} \approx 1.16 z_{0}$

which is precisely the ratio required to make $\gamma=0$ in a hyperbolic trap [9]. Notice also in Fig. 5 that in the limit $\Delta z_{\mathrm{c}} \rightarrow 0$, the ratio $\rho_{0} / z_{0}$ required to make $\gamma=0$ goes to the limiting value which makes $D_{4}=0$ for the simple trap of Fig. 1. To see how this comes about, observe that $V_{0} \partial D_{k} / \partial V_{c}$ and its first derivative with respect to $\Delta z_{\mathrm{c}}$ both vanish as $\Delta z_{\mathrm{c}} \rightarrow 0$. Setting the second derivative of $V_{0} \partial D_{2} / \partial V_{\mathrm{c}}$ equal to zero (to make $\gamma=0$ in the limit $\Delta z_{\mathrm{c}} \rightarrow 0$ ) produces the same condition on $\rho_{0} / z_{0}$ as does setting $D_{4}$ [of Eq. (12)] equal to zero for the trap of Fig. 1. 
Mechanical imperfections in $\rho_{0} / z_{0}$ for laboratory traps with cylindrical and hyperbolic electrodes will, of course, prevent $\gamma=0$ from being realized exactly. For cylindrical traps constructed to have $\gamma=0$ (by choosing $\rho_{0} / z_{0}$ from Fig. 5)

$$
\frac{\partial \gamma}{\partial\left[\rho_{0} / z_{0}\right]} \approx 2.66
$$

to within $1 \%$ for $\Delta z_{\mathrm{c}} / z_{0}$ ranging from 0 to $1 / 2$. A realizable imprecision of $10^{-3}$ in $\rho_{0} / z_{0}$, for example, thus means that $|\gamma| \approx 2 \times 10^{-3}$, which is still very much better than the $|\gamma| \approx 0.56$ for asymptotically symmetric traps with hyperbolic electrodes. The orthogonalized (i.e. $\gamma=0$ ) traps with hyperbolic electrodes are more sensitive to imprecisions in $\rho_{0} / z_{0}$ than are the cylindrical traps. For example, the hyperbolic trap with $\gamma=0$ described in Fig. 13 of ref. 9 has

$$
\frac{\partial \gamma}{\partial\left[\rho_{0} / z_{0}\right]} \approx 20
$$

which is a factor of seven more sensitive to imprecisions in $\rho_{0} / z_{0}$.

The tunabilities $V_{0} \partial D_{k} / \partial V_{\mathrm{c}}$ for $k>2$ are typically several orders of magnitude greater for cylindrical traps than for hyperbolic traps because the compensation potential is not so severely screened by the endcap and ring electrodes. As a result, anharmonicity compensation in a cylindrical trap differs from a hyperbolic trap in that a much smaller change in the compensation potential is required to change $D_{4}$ by a given amount. Figure 6 shows $V_{0} \partial D_{4} / \partial V_{c}$ for cylindrical traps with $\gamma=0$ as a function of the height of the compensation electrode, $\Delta z_{\mathrm{c}} / z_{0}$. For $\Delta z_{\mathrm{c}}=0$, this tunability vanishes as it must. By $\Delta z_{\mathrm{c}} / z_{0}=0.3$, however, $V_{0} \partial D_{4} / \partial V_{\mathrm{c}}$ is already equal to -0.18 or several orders of magnitude larger (in magnitude) than is typical for hyperbolic traps. To tune this particular cylindrical trap to make $\left|\tilde{D}_{4}\right|<10^{-4}$ (as has been accomplished in compensated traps with hyperbolic electrodes) thus requires an adjustment of $V_{c} / V_{0}$ to within $5 \times 10^{-4}$ of its optimum value. Figure 6 also shows $V_{0} \partial D_{6} / \partial V_{c}$. Although this tunability is also much larger than for hyperbolic traps, the ratio of $V_{0} \partial D_{6} / \partial V_{c}$ to $V_{0} \partial D_{4} / \partial V_{c}$ is approximately the same.

The $D_{k}^{(0)}$ for a cylindrical trap are also much larger than those calculated for a perfect hyperbolic trap because the trap electrodes are much less along equipotentials of the desired quadrupole potential. For small enough energy $E_{z}$, in the axial oscillation, only $D_{4}^{(0)}$ is important and it can be compensated. The required compensation potential to make $D_{4}=0$ in Eq. (4) is given by the ratio of $-D_{4}^{(0)}$ and $V_{0} \partial D_{4} / \partial V_{c}$. This ratio is plotted in Fig. 7. The required compensation potential is thus approximately given by $V_{c} / V_{0} \approx$ $-1 / 3$, which is still conveniently between the endcap and ring potentials. 


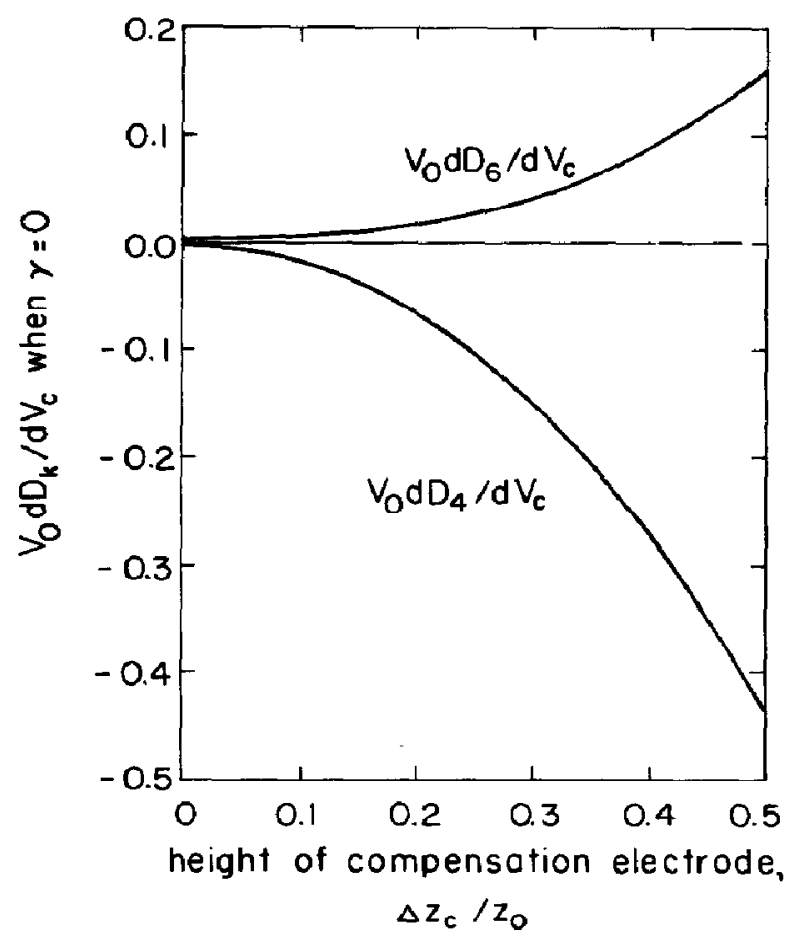

Fig. 6. The tunabilities $V_{0} \partial D_{4} / \partial V_{c}$ and $V_{0} \partial D_{6} / \partial V_{c}$ as a function of $\Delta z_{c} / z_{0}$ for the trap of Fig. 2 when $\rho_{0} / z_{0}$ is chosen (from Fig. 5) to make $\gamma=0$.

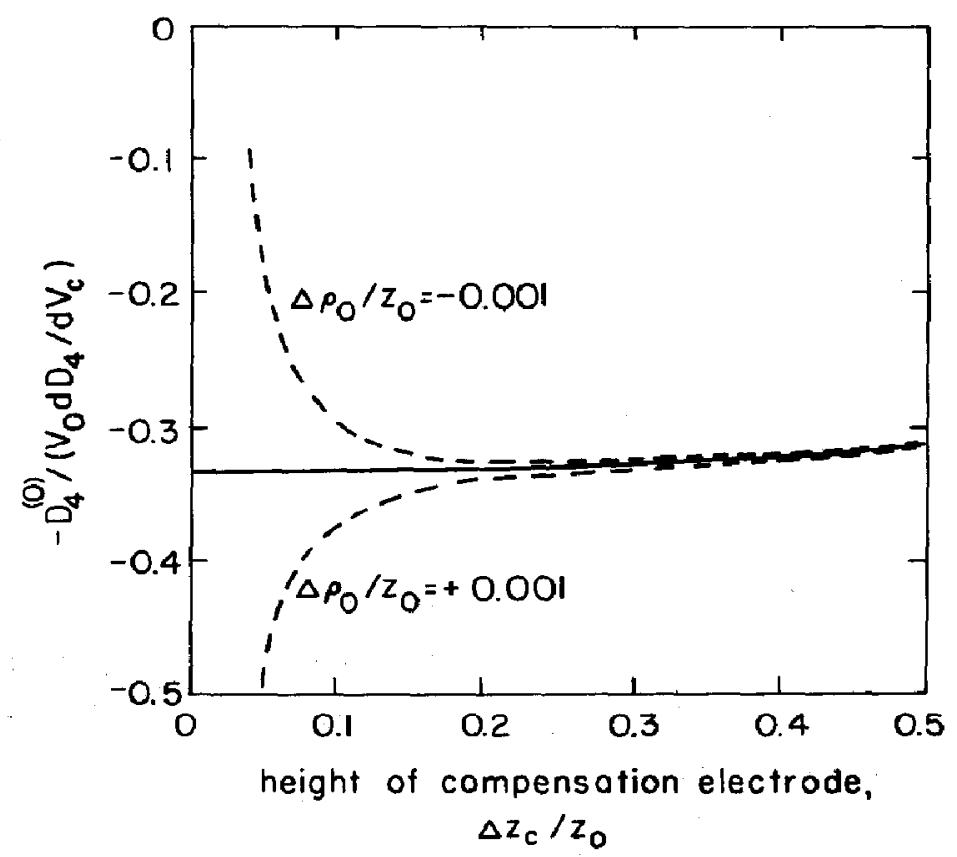

Fig. 7. The ratio of $-D_{4}^{(0)}$ to $V_{0} \partial D_{4} / \partial V_{\mathrm{c}}$ as a function of $\Delta z_{\mathrm{c}} / z_{0}$ for the trap of Fig. 2 when $\rho_{0} / z_{0}$ is chosen (from Fig. 5) to make $\gamma=0$ (solid lines) and for $\rho_{0} / z_{0}$ slightly larger and smaller than these values (broken lines). For perfectly aligned electrodes with no holes, slits, or imperfections, the vertical scale is the normalized compensation potential $V_{\mathrm{c}} / V_{0}$ required to make $D_{4}$ vanish. 
The broken lines are included in Fig. 7 to emphasize the sensitivity to imprecisions in $\rho_{0} / z_{0}$ for thin compensation electrodes. They represent departures of $\pm 10^{-3}$ from the value of $\rho_{0} / z_{0}$ required to make $\gamma=0$ and are not very important for $\Delta z_{\mathrm{c}} / z_{0}>0.2$.

Figure 7 must be interpreted carefully because misalignments, holes, slits and imperfections modify $D_{4}^{(0)}$ (and higher order $D_{k}^{(0)}$ ) so that the plotted values must be regarded as limiting values to be approached in the limit of a perfect cylindrical trap. As an example, one hyperbolic trap tuned at $\left|V_{\mathrm{c}} / V_{0}\right| \approx 10$, which corresponds to $\left|D_{4}^{(0)}\right| \approx 0.04$. Another was dissembled and reassembled and $D_{4}^{(0)}$ changed by approximately 0.003 in the process, presumably because of different electrode alignment. In both cases, the calculated values of $D_{4}^{(0)}$ were much smaller [9]. We must expect similar variations from the values plotted in Fig. 7 for cylindrical traps. The perfect trap estimates could be much better than was the case for hyperbolic traps, however, because the calculated $D_{4}^{(0)}$ is several orders of magnitude greater and thus might even dominate the contributions from imperfections. Even if it turns out that $D_{4}^{(0)}$ cannot be predicted accurately (as for hyperbolic traps), it will still be possible to tune $V_{\mathrm{c}}$ to make $D_{4}=0$. For small $E_{z}$, cylindrical traps are as good as hyperbolic traps.

For larger axial excitations, the contributions of $D_{6}$ to $\Delta \omega_{z}$ [as given in Eq. (5)] must also be considered. These contributions are quadratic in $E_{z} / m \omega_{z}^{2} d^{2}$. For a compensation potential which makes $D_{4}=0$, these contributions are substantial, as may be seen from Fig. 8. Fortunately, for a single trapped particle, the effect of $D_{6}$ may also be compensated by a slight additional adjustment of the compensation potential. To get a rough idea of how this comes about, consider the compensation potential which makes $\Delta \omega_{z}=0$ in Eqs. (4) and (5).

$\left[\frac{V_{\mathrm{c}}}{V_{0}}\right]_{\tilde{D}_{4}=0} \approx \frac{-D_{4}^{(0)}}{V_{0}\left(\partial D_{4} / \partial V_{\mathrm{c}}\right)}\left[1+{ }_{2}^{5} D \frac{E_{z}}{m \omega_{z}^{2} d^{2}}\right]$

The leading term is just the ratio plotted in Fig. 7 and discussed above. The factor in square brackets represents a small amplitude dependence in the compensation potential required to minimize amplitude-dependent shifts in $\omega_{z}$. The constant $D$ is given by

$D=\frac{D_{6}^{(0)}}{D_{4}^{(0)}}-\frac{V_{0}\left(\partial D_{6} / \partial V_{c}\right)}{V_{0}\left(\partial D_{4} / \partial V_{c}\right)}$

and Eq. (26) presupposes that both terms comprising $D$ are not large. In Fig. $9, D$ is plotted and is of order unity for $\Delta z_{c} / z_{0}>0.2$. Even for an imperfect trap for which $D_{4}^{(0)}$ and $D_{6}^{(0)}$ differ substantially from the values for a perfect trap, it may be reasonable to suppose that their ratio, and hence $D$, will not 

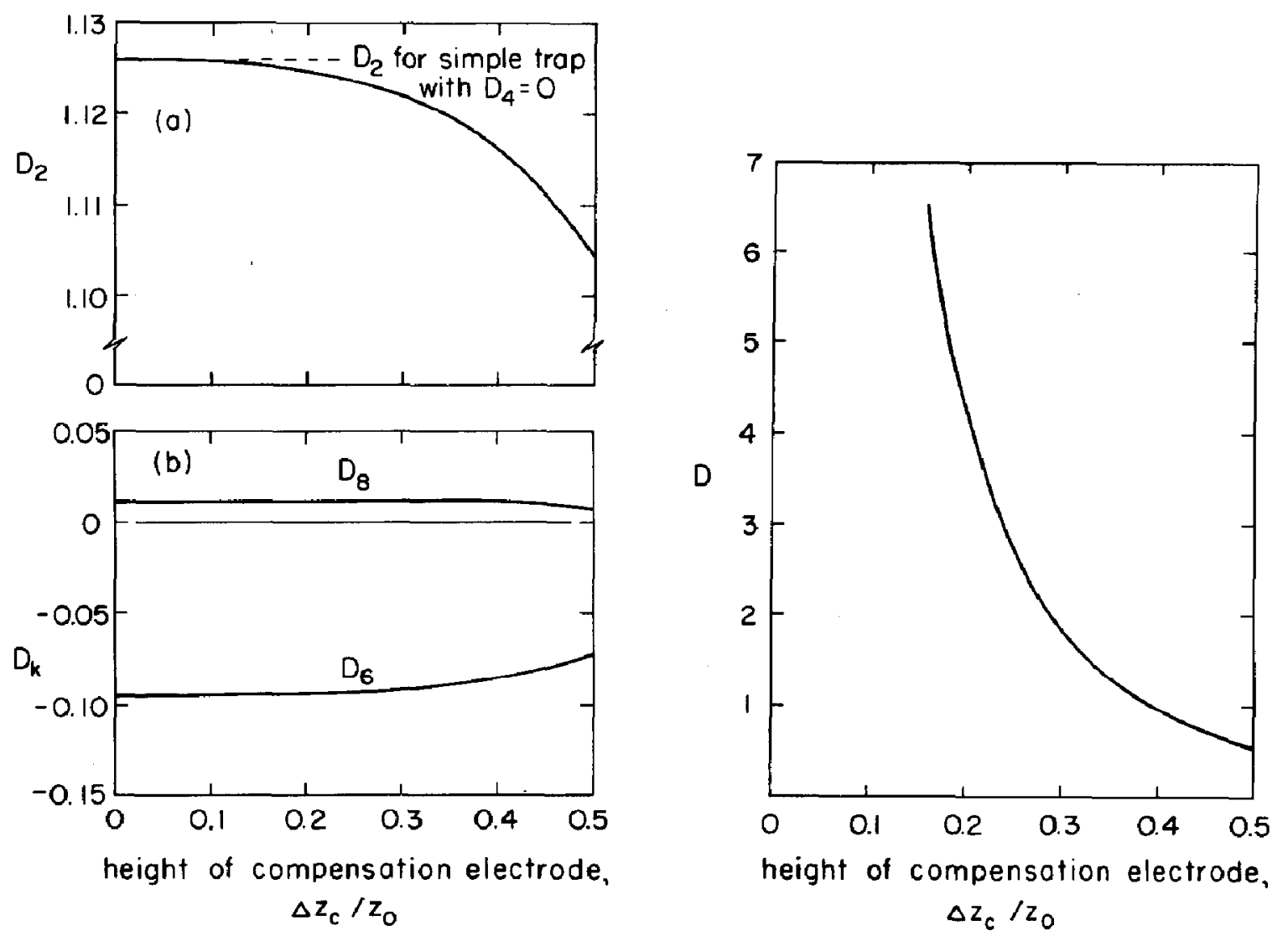

Fig. 8. The net $D_{4}, D_{6}$ and $D_{8}$ for the trap of Fig. 2 when $\rho_{0} / z_{0}$ as chosen (from Fig. 5) to make $\gamma=0$ and the value of $V_{c} / V_{0}$ (from Fig. 7) is applied to make $D_{4}=0$.

Fig. 9. The constant $D$ which describes the amplitude dependence of the compensation potential which is required to minimize anharmonicities.

differ substantially from unity. For a single particle with $E_{z} / m \omega_{z}^{2} d^{2}$ of order $10^{-3}$ as claimed in ref. 3 , the amplitude dependence should be very small.

The scenario presented for compensating $D_{6}$ may well be too optimistic. Trap imperfections which are not symmetric under $z \rightarrow-z$ and thermal noise potentials will certainly complicate matters. While perfect hyperbolic electrodes would certainly provide a larger harmonic trapping volume than would perfect cylindrical trap electrodes, there is growing evidence that holes, slits, misalignments and imperfections are presently limiting the harmonic trapping volume for existing hyperbolic traps. To what extent this is true, and thus to what extent a cylindrical trap will be useful for precision work, must now be tested experimentally. 


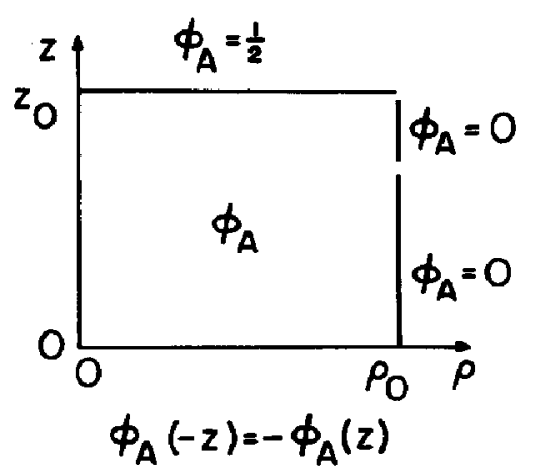

Fig. 10. Boundary conditions which uniquely determine the solution to Laplace's equation $\phi_{A}$ along with axial symmetry about the $z$ axis and antisymmetry under the reflection $z \rightarrow-z$.

\section{DAMPING AND DRIVING THE AXIAL RESONANCE}

Damping and driving the axial oscillation was discussed earlier by Wineland and Dehmelt [15]. More recently, a relaxation calculation was used to calculate the key constants for hyperbolic traps [10]. Rather than repeat these discussions, we shall simply state the results for cylindrical traps. They do not differ substantially from the hyperbolic case.

The required solution to Laplace's equation is called $\phi_{\mathrm{A}}$ as in ref. 10 . It is defined uniquely by its boundary conditions in Fig. 10 and by its antisymmetry under reflections $z \rightarrow-z$. For small $r / z_{0}$

$\phi_{\mathrm{A}}=\frac{1}{2} \sum_{k_{\text {odd }}=1}^{\infty} B_{k}\left[\frac{r}{z_{0}}\right]^{k} P_{k}(\cos \theta)$

As in the earlier paper [10], distances are scaled here by $z_{0}$ rather than by $d$ because for large $\rho_{0} / z_{0}$ the solution $\phi_{\mathrm{A}}$, and hence the $B_{k}$, approach limiting values which are independent of $\rho_{0}$.

The lowest order coefficient, $B_{1}$, is certainly the most important for particles trapped near the center of the trap, where $r / z_{0} \ll 1$. In this region, $\phi_{\mathrm{A}} \approx B_{1} z / 2 z_{0}$ so that $B_{1}$ is the measure of a uniform electric field at the center of the trap. A small potential, $V_{D C}$, applied across the endcaps produces an axial force $\partial\left(q V_{\mathrm{DC}} \phi_{\mathrm{A}}\right) / \partial z$ on a trapped particle. The potential $V_{\mathrm{DC}}$ can be oscillatory as long as its wavelength is much larger than the trap dimensions. One consequence is that a small static potential $V_{\mathrm{DC}}$ applied across the endcaps moves the center of the axial oscillation from $z=0$ to the metastable equilibrium value of

$z_{\mathrm{e}}=\frac{d^{2}}{2 z_{o}} B_{1} \frac{q V_{\mathrm{DC}}}{m \omega_{z}^{2} d^{2}}$ 
Such potentials have been used in hyperbolic traps. A resistor, $R$, connected between the endcaps will damp the axial oscillation with damping constant $[10,15]$.

$\Gamma_{z}=\frac{1}{m}\left[\frac{q B_{1}}{2 z_{0}}\right]^{2} R$

Notice that $\Gamma_{2}$ goes as the square of $B_{1}$.

An explicit expression for the $B_{k}$ is easy to obtain by solving for $\phi_{\mathrm{A}}-z / 2 z_{0}$ using the standard techniques reviewed in Sect. 3. The result is (for $k>0$ and odd)

$B_{k}=\delta_{k, 1}+\frac{2(i \pi)^{k-1}}{k !} \sum_{n=1}^{\infty} \frac{n^{k-1}}{J_{0}\left[i n \pi p_{0} / z_{0}\right]}$

The $B_{k}$ are easily evaluated. The first three are plotted in Fig. 11 as a function of a much wider range of $\rho_{0}^{2} / 2 z_{0}^{2}$ than is useful for trap design so that limiting values are clearly exhibited. For large $\rho_{0} / z_{0}, B_{1}$ goes to 1 and all other $B_{k}$ vanish. This corresponds to a constant electric field between two infinitely extended capacitor plates. As the ring is pulled in toward the center of the trap, electric field lines increasingly terminate on the ring rather than penetrating through the center of the trap so that $B_{1}$ decreases. Then, $B_{3}$ and $B_{5}$ increase in magnitude to compensate. For smallest $\rho_{0} / z_{0}$, exponential screening sets in and all the $B_{k}$ go rapidly to zero.

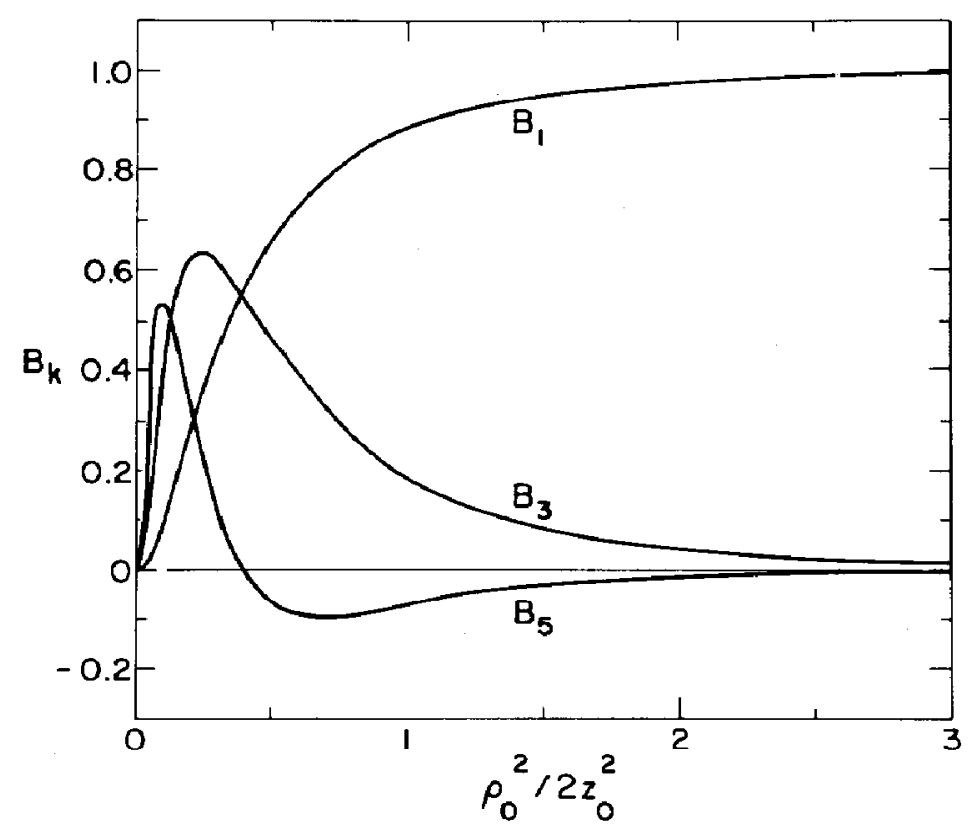

Fig. 11. Lowest-order expansion coefficient $B_{k}$ for $\phi_{\mathrm{A}}$ as a function of $\rho_{0}^{2} / 2 z_{0}^{2}$. 


\section{CONCLUSION}

Penning traps with flat endcap and cylindrical ring electrodes are shown to be promising alternatives to the existing traps with hyperbolic electrodes, even for precision work. The electrostatic potential within such traps is calculated using standard series solutions to Laplace's equation. Coefficients which are important for particle trapping are discussed and plotted to facilitate trap design.

An important new result is that a judicious choice of the relative dimensions of the electrically compensated, cylindrical trap makes the axian oscillation frequency of a trapped particle completely independent of adjustments in the potential applied to the compensation electrodes. Such adjustments are required to make the motion of the particle more harmonic. The "magic" geometry which produces such orthogonalized anharmonicity compensation has now been found for hyperbolic and cylindrical traps. It has also become clear that such orthogonalized anharmonicity compensation will be possible for other trap configurations as well, to permit special access to the center of the trap, though numerical computations will generally be required to identify the "magic" dimensions.

\section{REFERENCES}

1 D. Wineland, P. Ekstrom and H. Dehmelt, Phys. Rev. Lett., 31 (1973) 1279.

2 Reviewed in H. Dehmelt, 1982/83 McGraw-Hill Yearbook of Science and Technology, McGraw-Hill, New York, 1982; P. Ekstrom and D. Wineland, Sci. Am., 243 (Aug.) (1980) 105.

3 R.S. Van Dyck, Jr., P.B. Schwinberg and H. Dehmelt, in B. Kursuhoglu, A. Perlmutter and L. Scott (Fds.), New Frontiers in High Energy Physics, Plenum Press, New York, 1978; R.S. Van Dyck, Jr., P.B. Schwinberg and H.G. Dehmelt. Bull. Am. Phys. Soc., 24 (1979) 758.

4 P.B. Schwinberg, R.S. Van Dyck, Jr. and H.G. Dehmelt, Phys. Rev. Lett., 47 (1981) 1679.

5 T. Kinoshita and W.B. Lindquist, Phys. Rev. Lett., 47 (1981) 1573.

6 R.S. Van Dyck, Jr. and P.B. Schwinberg, Phys. Rev. Lett., 47 (1981) 395; R.S. Van Dyck, Jr., F.L. Moore and P.B. Schwinberg, Bull. Am. Phys. Soc., 28 (1983) 791.

7 G. Gabrielse and H. Dehmelt, Natl. Bur. Stand. (U.S.) Spec. Publ. 617, 1981.

8 R.S. Van Dyck, Jr., D. Wineland, P. Ekstrom and H. Dehmelt, Appl. Phys. Lett., 28 (1976) 446.

9 G. Gabrielse, Phys. Rev. A, 27 (1983) 2277.

10 G. Gabrielse, Phys. Rev. A, 29 (1984).

11 F.M. Penning, Physica, 3 (1936) 873.

12 One dimensional anharmonic oscillators are discussed in L.D. Landau and E.M. Lifshitz, Mechanics, translated by J.S. Bell, Pergamon Press, New York, 1976, pp. 87-89.

13 Trap designed by $F$. Walls and reported in ref. 8.

14 Trap designed by $G$. Gabrielse and reported in ref. 7.

15 D. Wineland and H. Dehmelt, J. Appl. Phys., 46 (1975) 919. 